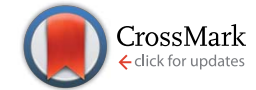

Cite this: RSC Adv., 2017, 7, 8394

Received 4th November 2016 Accepted 19th January 2017

DOI: 10.1039/c6ra26330e

www.rsc.org/advances

\section{First step towards a model system of the drug delivery network based on amide-POSS nanocarriers $\dagger$}

\begin{abstract}
Łukasz John, ${ }^{*}$ Mariola Malik, Mateusz Janeta and Sławomir Szafert
Among the varied and diverse family of drug delivery systems (DDSs), such as polymer-drug conjugates, polymeric micelles, dendrimers and molecular containers, polyhedral oligomeric silsesquioxanes (POSS) occupy a special position within the group of biocompatible drug carriers. The extraordinary recommendation of POSS for such systems derives from their three-dimensional structure, nanoscale size, low toxicity, and efficient cellular uptake. These conjugates can be strictly designed compared for instance to micelles, dendrimeric-drug or polymer/inorganic hybrid composites, in which the amount of therapeutics attached to the carrier is rather random, and there is a high probability that some of the drug molecules can be completely trapped inside the ramified structures. The combination of fully functionalized amide-POSS and pharmaceuticals might create a so-called sophisticated DDS. In such a system, the adsorbed drug molecules can be released under physiological conditions and then the POSS-based carrier will be hydrolyzed (at $\mathrm{pH}=7.40$ ) to a non-toxic carboxylic acid salt and a water soluble polyhedral oligomeric silsesquioxane containing an aminopropyl group that can be safely removed from the organism.
\end{abstract}

\section{Introduction}

Controlled release systems have continuously been developed to deliver a precise amount of a drug, to increase the effect of the drug on the body, to protect it from physiological degradation, to improve patient comfort and to be able to control the location of drug delivery. ${ }^{1}$ In this case, the bottom-up approach is strictly based on nanobuilding blocks possessing fine-tuned properties. $^{2,3}$ For instance, in the past decade, a versatile family of three-dimensional nanoblocks has been formed by various molecular nanoparticles (MNPs) including polyoxometalates, fullerenes, polyhedral oligomeric silsesquioxanes (POSS) and folded proteins., ${ }^{4,5}$ A well-functionalized MNP surface that possesses reactive groups located in well-thoughtout places allows a distinct self-assembly behavior and leads to sophisticated material properties. ${ }^{3}$ This phenomenon was inter alia demonstrated in the formation of so-called FrankKasper phases from asymmetric shape-persistent nanosized giant tetrahedra constructed by placing different POSS-based molecular nanoparticles at the vertices of a rigid tetrahedral framework. ${ }^{6}$ In the literature, there can be found many possibilities that allow drug attachment to the surface of

Faculty of Chemistry, University of Wroctaw, 14 F. Joliot-Curie, 50-383 Wrocław, Poland.E-mail: lukasz.john@chem.uni.wroc.pl

$\dagger$ Electronic supplementary information (ESI) available: ${ }^{1} \mathrm{H}$ and ${ }^{13} \mathrm{C}$ NMR, FT-IR, EDS spectra, TG-DTA, DSC, PXRD patterns and calibration curves for acetaminophen and ibuprofen. See DOI: 10.1039/c6ra26330e nanoparticles, such as adsorption, covalent bonding and electrostatic interactions. In our previous studies, we have shown that octa functionalized derivatives of POSS have a strong tendency to form supramolecular networks and are able to trap small molecules in a crystal lattice (Fig. 1). Here, depending on the nature of substituent, POSS species form star-like (for a phenyl group) or rod-like (for a $p$-halogen substituted phenyl group) structures. ${ }^{7}$

Well-designed drug delivery systems are extremely difficult to estimate and design. This is due to the fact that there is

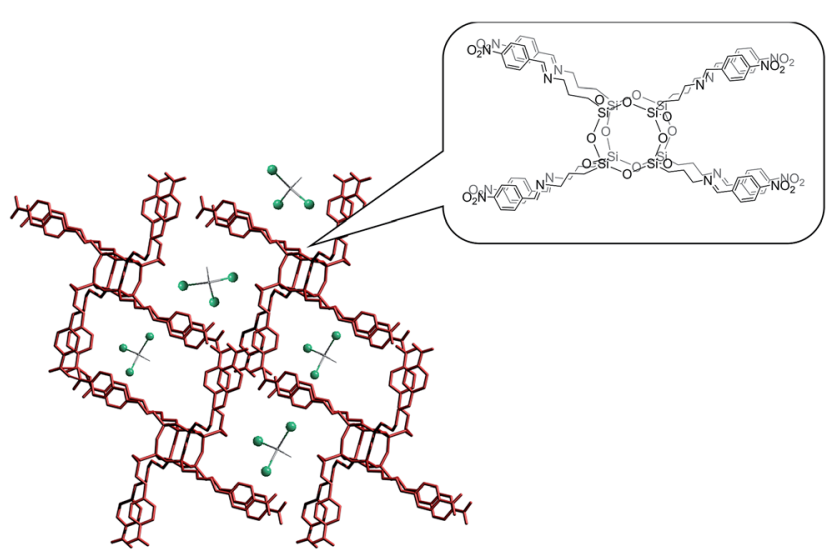

Fig. 1 View of the 3D interpenetrated network in the crystal structure of $p$-nitrophenyl-substituted POSS. Between the side arms, small molecules (like chloroform or methanol) can be trapped. 
a number of complex mechanisms that are involved in the release process of pharmacologically active compounds. ${ }^{1}$ In such systems, some crucial factors should be taken into consideration, such as the degree of adsorption of the drug and diffusion limitations in the medium that seems the most effective way of treatment. The rapid development of nanotechnology has greatly affected many areas of science, including drug delivery systems that offer time-dependent controlled release from the carrier, targeted delivery and are site-specific. ${ }^{8}$ In this field, many advantages over the conventional delivery systems are offered by nanomaterials, including micelles, nanocapsules and nanoparticles. Moreover, such an approach makes it possible to omit various drawbacks strictly associated with direct drug delivery, such as poor drug solubility, irritation of the stomach epithelium, poor biodistribution, lack of selectivity and rapid breakdown in vivo. ${ }^{1,9}$ The physicochemical properties of such nanostructured drug delivery systems are tuned based on either the drug type or the delivery route such as oral, nasal or transdermal.

Nanomedicine is a term that covers the broad application of nanotechnology in the human healthcare. It gives rise to a magnitude of possibilities to use particulate nanomaterials with dimensions within the 1-100 $\mathrm{nm}$ range, which corresponds to the major dimension of the particles in the body, antibodies, membrane receptors, nucleic acids, proteins, etc. This feature creates a broad spectrum of different options for the functionalization of nanoparticles at the cellular level, which can support a range of medical activities such as the precise diagnosis of diseases, imaging or topical drug therapy ${ }^{10}$ In this aspect, polyhedral oligomeric silsesquioxanes provide enormous possibilities. Cubic ( $\mathrm{T}_{8}$-type) cage-like silsesquioxanes are an organosilicon species with the general formula $\left(\mathrm{RSiO}_{3 / 2}\right)_{8}$ (where $\mathrm{R}=\mathrm{H}$ or a wide variety of organic groups) which adopt structures with siloxane $\mathrm{Si}-\mathrm{O}-\mathrm{Si}$ linkages and tetrahedral silicon vertices, where diverse organic moieties, including drugs, can be effectively anchored. ${ }^{11}$ For example, Rotello et al. reported on a controlled drug delivery system based on POSS units. ${ }^{12}$ They proved that functionalized polyhedral oligomeric silsesquioxanes exhibit very low toxicity and efficient uptake in the cytoplasm of Cos-1 cells and demonstrated that the conjugation of a small molecule either directly to octa-ammonium-POSS or through a cleavable linker enables the delivery of drugs that are insoluble in water or exhibit low cellular uptake. Also star-shaped poly(benzyl L-aspartate)-blockpoly(ethylene glycol) copolymers with POSS core constitute potential carriers for drug delivery. ${ }^{13}$ In turn, Kaneshiro et al. reported on the globular morphology, the highly functionalized surface and the rigid structure of octa(3-aminopropyl) silsesquioxane-based poly(L-lysine) nanoglobules, which are able to form conjugates with a large number of $\mathrm{Gd}(\mathrm{III})$ chelates and serve as efficient MRI contrast agents. ${ }^{14}$

From this point of view, POSS constitute attractive building blocks for drug delivery platforms as biocompatible drug carriers due to their three-dimensional structure, nanoscale size, low toxicity, efficient cellular uptake and high solubility. ${ }^{10,15}$ It should also be emphasized that silica-based materials are generally widely used in the field of biomedicine because of their chemical inertness and biocompatibility. ${ }^{16}$ In this manner, silica nanoparticles have been developed for their use in magnetic resonance imaging, ${ }^{17}$ the delivery of antisense oligonucleotides, ${ }^{18}$ and the delivery of drugs such as dexmedetomidine ${ }^{19}$ and cefradine. ${ }^{20}$ Therefore, nanocarriers based on cage-like silsesquioxanes form attractive alternative systems, with the ability to create more interesting functionalities in respect of binding pharmacological agents. It should also be noted that a drug-POSS system can be strictly designed in comparison to, e.g., micelles/dendrimeric-drug or polymer/ inorganic hybrid nanoparticles, in which the amount of the pharmaceutical active species that are bound is rather statistic/ random and there is a serious danger that some quantity of the drug will be completely trapped inside the crowded structures by the undesired "encapsulation" effect, which will prevent efficient drug release. ${ }^{21}$ The core-shell architecture of POSS is essential for applications as carriers to trap various organic molecules. This is achieved thanks to multivalency, which characterizes some nanosystems, such as hyperbranched polymers, micelles or dendrimers, which contain a number of functional, ramified/sizable side-chains.

In fact, many studies need to be performed to get a better understanding of the impact of various parameters on drug attaching efficiency. ${ }^{22}$ Despite promising results achieved for such drug delivery systems (hyperbranched polymers, dendrimers, micelles), there is a constant search for simpler, more effective and cheaper solutions. Being inspired by the excellent biocompatibility of functionalized POSS, ${ }^{23}$ in this work we present a "simpler", but efficient, delivery system based on amido-functionalized POSS, which are expected to be attractive carriers for nonsteroidal anti-inflammatory drugs (NSAIDs), such as ibuprofen and acetaminophen. In this paper, we present synthesis and release aspects based on model studies on known and used anti-inflammatory drugs. In the nearest future, the materials described here will be used as additive agents in the formation of improved three-dimensional scaffolds based on silane/methacrylate-based organic-inorganic composites. $^{24,25}$

\section{Experimental}

\subsection{General procedures and chemicals}

Syntheses of 1-3 were carried out under $\mathrm{N}_{2}$ with the use of standard Schlenk techniques. Benzoyl chloride (98\%, Aldrich), 4-nitrobenzoyl chloride ( $98 \%$, Aldrich), 4-fluorobenzoyl chloride (98\%, Aldrich), (3-aminopropyl)triethoxysilane (99\%, Aldrich), triethylamine (99.5\%, Aldrich), dimethyl sulfoxide- $\mathrm{d}_{6}$ (99.9 atom\% D, Aldrich), HCl (36-38\%, Avantor Performance Materials Poland S.A.) and ethanol (anhydrous, J. T. Baker) were used without further purification unless stated otherwise. Dimethylformamide (99.8\% anhydrous, Aldrich) was dried over activated $4 \AA$ molecular sieves prior to use. Acetaminophen (99\%, Aldrich) and ibuprofen sodium salt (98\%, racemic mixture, Aldrich) were predried in vacuo $\left(25{ }^{\circ} \mathrm{C}, 0.5 \mathrm{mbar}\right)$. $\mathrm{Na}_{2} \mathrm{HPO}_{4} \cdot 12 \mathrm{H}_{2} \mathrm{O}$ (analytical grade, Avantor Performance Materials Poland S.A.) and $\mathrm{NaH}_{2} \mathrm{PO}_{4} \cdot 2 \mathrm{H}_{2} \mathrm{O}$ (analytical grade, Chempur) were used without further purification. Aqueous 
solutions were prepared with doubly distilled water. Phosphate buffer solutions $0.10 \mathrm{M}$ with $\mathrm{pH}=7.40 \pm 0.02$ were prepared by mixing aqueous stock solutions of $\mathrm{Na}_{2} \mathrm{HPO}_{4} \cdot 12 \mathrm{H}_{2} \mathrm{O}$ and $\mathrm{NaH}_{2} \mathrm{PO}_{4} \cdot 2 \mathrm{H}_{2} \mathrm{O}$. Buffers were stored at $4{ }^{\circ} \mathrm{C}$ and warmed up to $37{ }^{\circ} \mathrm{C}$ before being used.

\subsection{Methods}

The ${ }^{1} \mathrm{H}$ NMR and ${ }^{13} \mathrm{C}$ NMR spectra were recorded using a Bruker Avance 500 spectrometer. ${ }^{1} \mathrm{H}$ NMR spectra were collected at $500.13 \mathrm{MHz}$. Spectra were referred to the residual solvent signal (DMSO- $\left.\mathrm{d}_{6} 2.50\right)$ as an internal reference. ${ }^{13} \mathrm{C}$ NMR spectra were collected at $125.8 \mathrm{MHz}$ and referred to the solvent signal $\left(\left({ }^{13} \mathrm{CH}_{3}\right)_{2} \mathrm{SO} 39.52\right)$. Fourier-Transform Infrared Spectra (FTIR) were recorded on a Bruker Vertex 70 FTIR spectrometer in the transmission mode. Data were acquired in the $4000-400 \mathrm{~cm}^{-1}$ range with a precision of $\pm 2 \mathrm{~cm}^{-1}$. Sample spectra were recorded as $\mathrm{KBr}$ pellets. Optical grade, random cuttings of $\mathrm{KBr}$ were ground, with $1.0 \mathrm{wt} \%$ of the sample to be analyzed and pressed $\mathrm{KBr}$ pellets. Thermogravimetry and differential thermal analyses (simultaneous TG-DTA) were recorded with a Setaram SETSYS 16/18 instrument. The calibrations of temperature and weight were performed using indium (NIST SRM 2232) as the standard material for temperature and a set of exactly weighed samples supplied by Shimadzu for weight. Samples for thermogravimetric characterization were placed in open alumina crucibles in synthetic air $\left(60 \% \mathrm{~N}_{2}, 40 \% \mathrm{O}_{2}\right)$, flow rate: $1 \mathrm{dm}^{3} \mathrm{~h}^{-1}$. A heating rate of $10{ }^{\circ} \mathrm{C} \mathrm{min}^{-1}$ was applied and all samples were studied between 30 and $1000{ }^{\circ} \mathrm{C}$. Differential Scanning Calorimetry (DSC) traces were obtained using a Perkin Elmer model 8500. Temperature and enthalpy calibrations were performed using high purity standards ( $n$-heptane and indium). The samples (3-5 mg) were placed in sealed Al-pans at a heating rate of $10{ }^{\circ} \mathrm{C} \mathrm{min}^{-1}$. The temperature range for samples with ibuprofen was from $30{ }^{\circ} \mathrm{C}$ to $120{ }^{\circ} \mathrm{C}$, and for samples with acetaminophen, from $30{ }^{\circ} \mathrm{C}$ to $200{ }^{\circ} \mathrm{C}$. Samples for transmission electron microscopy (TEM) were prepared by dispersing the powder in hexane and depositing the suspension on a holed carbon copper grid. The images were obtained with a FEI Tecnai G2 F20 X-TWIN Transmission Electron Microscope equipped with a Penta FET EDX detector. Powders were characterized recording X-ray powder diffraction (XRD) patterns with a Bruker D8 ADVANCE diffractometer equipped with a copper lamp $\left(\lambda_{\mathrm{CuK} \alpha}=1.5418 \AA\right)$. Standard measurements were done for $2 \theta=$ $10-50^{\circ}$ with a $2 \theta$ step of $0.008^{\circ}$ and a counting time of $1 \mathrm{~s} . \mathrm{pH}$ values were measured using a Lutron $\mathrm{PH}-207 \mathrm{pH}$ meter equipped with a EuroSensor combination electrode calibrated with Avantor Performance Materials Poland S.A. standard buffer solutions of $\mathrm{pH} 4.00 \pm 0.05$, and $\mathrm{pH} 10.00 \pm 0.05$. The UV/Vis absorption spectra were recorded at room temperature with a double beam Cary 500 SCAN UV-VIS-NIR spectrophotometer. Measurements were performed on a $3.5 \mathrm{~mL}$ quartz cell with an optical path length of $10 \mathrm{~mm}$. The optimized operating conditions were: spectra range 200 to $450 \mathrm{~nm}$ at intervals of $0.5 \mathrm{~nm}$, scan speed $150.0 \mathrm{~nm} \mathrm{~min}^{-1}$, ave. time $0.2 \mathrm{~s}$, bandwidth $0.2 \mathrm{~nm}$. GC-FID and GC-MS spectra were obtained using an HP 5890 II (Hewlett Packard) instrument with an HP 5971A mass detector.
An HP 5 capillary column $(25 \mathrm{~m} \times 0.2 \mathrm{~mm})$ was used with a nonpolar liquid phase containing $95 \%$ of dimethyl- and $5 \%$ of diphenyl-polysiloxane, using helium as the carrier gas. Mass spectra were obtained using an HP 5971A mass selective detector. The particle size distribution of synthesized materials was determined by dynamic light scattering (DLS) by a Malvern ZETASIZER Nano ZS particle size analyzer equipped with diode laser $(4 \mathrm{~mW})$ operating at $633 \mathrm{~nm}$. All measurements were carried out at $25{ }^{\circ} \mathrm{C}$.

\subsection{Syntheses of 1-3}

Amido-functionalized polyhedral oligomeric silsesquioxanes 13 were obtained according to a previously reported procedure. ${ }^{26}$

2.3.1. 1: Benzoyl chloride (0.888 mL, $1.054 \mathrm{~g}, 7.50 \mathrm{mmol}, 8.8$ equiv.) was added dropwise to a solution of octa(3-aminopropyl) silsesquioxane $(1.00 \mathrm{~g}, 0.852 \mathrm{mmol})$ and $\mathrm{NEt}_{3}(2.20 \mathrm{~mL}, 1.154 \mathrm{~g}$, $15.75 \mathrm{mmol}, 18.5$ equiv.) in DMF ( $\left.40 \mathrm{~mL}, 0{ }^{\circ} \mathrm{C}\right)$. After stirring overnight, the crude product was precipitated by slow addition to $1 \mathrm{M}$ aqueous $\mathrm{HCl}\left(70 \mathrm{~mL}, 0{ }^{\circ} \mathrm{C}\right)$. Filtration, washing with cold saturated $\mathrm{NaHCO}_{3}(40 \mathrm{~mL})$, next with water, and drying in vacuo $\left(25{ }^{\circ} \mathrm{C}, 0.5 \mathrm{mbar}\right)$ gave a white solid $0.801 \mathrm{~g}$ (55\%). The spectroscopic data were in agreement with those in the literature.

2.3.2. 2: 4-Nitrobenzoyl chloride (1.392 g, $7.501 \mathrm{mmol}, 1.1$ equiv.) was added dropwise to a solution of octa(3-aminopropyl) silsesquioxane (1.00 g, $0.852 \mathrm{mmol})$ and $\mathrm{NEt}_{3}(2.20 \mathrm{~mL}, 1.596 \mathrm{~g}$, $15.75 \mathrm{mmol}, 18.5$ equiv.) in DMF (40 mL, $\left.0{ }^{\circ} \mathrm{C}\right)$. After stirring overnight, the crude product was precipitated by slow addition to $1 \mathrm{M}$ aqueous $\mathrm{HCl}\left(70 \mathrm{~mL}, 0^{\circ} \mathrm{C}\right)$. Filtration, precipitation with cold saturated $\mathrm{NaHCO}_{3}(40 \mathrm{~mL})$, washing with water and drying in vacuo $\left(25{ }^{\circ} \mathrm{C}, 0.5 \mathrm{mbar}\right)$ gave a white solid $1.574 \mathrm{~g}$ (89\%).

2.3.3. 3: 4-Fluorobenzoyl chloride $(0.800 \mathrm{~mL}, 1.037 \mathrm{~g}$, $6.77 \mathrm{mmol}, 8.8$ equiv.) was added dropwise to a solution of octa(3-aminopropyl)silsesquioxane $(0.903 \mathrm{~g}, 0.769 \mathrm{~mol})$ and $\mathrm{NEt}_{3}$ (1.98 mL, $1.439 \mathrm{~g}, 14.23 \mathrm{mmol}, 18.5$ equiv.) in DMF (40 mL, $0{ }^{\circ} \mathrm{C}$ ). After stirring overnight, the white crude product was precipitated by slow addition to $1 \mathrm{M}$ aqueous $\mathrm{HCl}\left(70 \mathrm{~mL}, 0{ }^{\circ} \mathrm{C}\right)$. Filtration, precipitation with cold saturated $\mathrm{NaHCO}_{3}(40 \mathrm{~mL})$, washing with water and drying in vacuo $\left(25^{\circ} \mathrm{C}, 0.5 \mathrm{mbar}\right)$ gave a white solid $0.760 \mathrm{~g}(48 \%)$.

\subsection{Preparation of drug-loaded nanoparticles}

Ibuprofen or acetaminophen were dissolved in ethanol to give solution of $6.99 \mathrm{mM}$ concentration. Appropriate amounts of amido-functionalized polyoctahedral oligomeric silsesquioxanes (1, $0.145 \mathrm{~g} ; 2,0.182 \mathrm{~g} ; 3,0.163 \mathrm{~g} ; 8.75 \times 10^{-2} \mathrm{mmol}$ ) were precisely weighed and then placed in vials filled with $10 \mathrm{~mL}$ of ethanolic drug solution. The suspension were stirred for $24 \mathrm{~h}$ at $37{ }^{\circ} \mathrm{C}$. The ibuprofen and acetaminophen-loaded POSS were separated from this solution by centrifugation and then dried under vacuum at $40{ }^{\circ} \mathrm{C}$.

\subsection{Nanoparticle entrapment efficiency}

Specific amounts of POSS nanoparticles were dispersed in drug ethanolic solution. The suspension of 1-3 with the appropriate drug was mixed for $48 \mathrm{~h}$ at $37{ }^{\circ} \mathrm{C}$ and then centrifuged. After that, the concentration of the drug in the supernatant was 
measured. The measurement was obtained by reading absorbance at the maximum of the spectrum $\left(\lambda_{\max }=221 \mathrm{~nm}\right.$ for ibuprofen, $\lambda_{\max }=245 \mathrm{~nm}$ for acetaminophen). The amount of the loaded drug was the difference between the total amount of the drug and the quantity in the supernatant. The entrapment efficiency was calculated as follows:

$$
\mathrm{EE}=\frac{W_{\mathrm{t}}-W_{\mathrm{d}}}{W_{\mathrm{t}}} \times 100 \%
$$

where: $W_{\mathrm{t}}=$ weight of the drug in the initial solution; $W_{\mathrm{d}}=$ weight of the free drug in solution after drug loading.

\subsection{In vitro drug release studies}

Vials with dried samples were filled with $10 \mathrm{~mL}$ of a phosphate buffer and stirred. After suitable time intervals $(0.5,1,1.5,2,2.5$, $3,4,5,10,12,24,48,60,96$, and $108 \mathrm{~h}$ counting from the beginning of experiment), $5 \mathrm{~mL}$ of the solution was taken and used for measurement. Then, a fresh portion of the phosphate buffer was added. Drug release profiles for the samples were determined by measuring the concentration of the released drugs in the phosphate buffer. The samples were kept in vials and stirred magnetically at $37{ }^{\circ} \mathrm{C}$ throughout the duration of the experiment. The release of the drug was determined by UV-Vis spectroscopy $\left(\lambda_{\max }=221 \mathrm{~nm}\right.$ for ibuprofen, $\lambda_{\max }=245 \mathrm{~nm}$ for acetaminophen). Initial drug samples for the calibration curve were prepared in the phosphate buffer: $10 \mathrm{mg}$ of each drug was dissolved in $100 \mathrm{~mL}$ of the phosphate buffer with $\mathrm{pH}=7.40 \pm$ 0.02 and well shaken. The other solutions of the drugs were prepared by dilution of the initial one. The concentrations of the

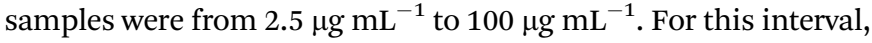
the calibration curve fits the Lambert and Beer law. Only matched cuvettes were used when obtaining the spectra. Released products were identified by GC-MS.

\section{Results and discussion}

\subsection{The aim of the study and the preparation of the carriers}

In this study, octa-functionalized amide-POSS were utilized as platforms for drug carriers. Our choice was strictly dictated by the nanosized architecture of polyhedral oligomeric silsesquioxanes $\left(\mathrm{RSiO}_{3 / 2}\right)_{8}$, which offer an opportunity for the modification of their $\mathrm{R}$ side chains through the addition of multiple organic groups tethered to their vertices and in consequence for the synthesis of systems that are able to trap various organic molecules such as drugs. In such a system, adsorbed drug molecules can be released under physiological conditions and then the POSS-based carrier will be hydrolyzed $(\mathrm{pH}=7.40)$ to a proper non-toxic carboxylic acid salt and a water soluble polyhedral oligomeric silsesquioxane containing an aminopropyl group - products which can be safely removed from the organism (Fig. 2).

Amido-functionalized compounds 1-3 were obtained via benzoylation reaction between octa(3-aminopropyl)silsesquioxane hydrochloride (OAS-POSS-Cl) and an appropriate acyl chloride in the presence of $\mathrm{NEt}_{3}$ and $N, N$-dimethylformamide (DMF) (Scheme 1).

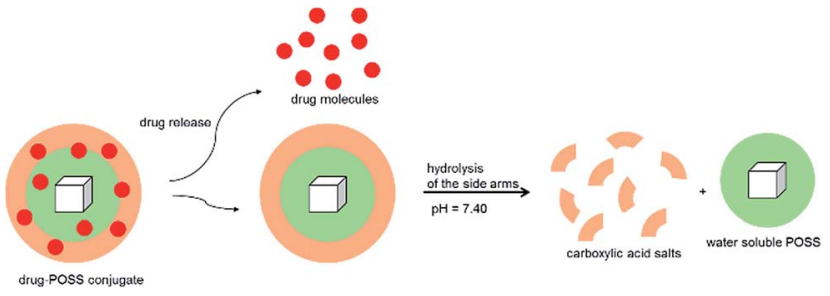

Fig. 2 Idea of a drug-POSS delivery system.

Benzoylation reactions were carried out using a previously reported method. ${ }^{26}$ The formation of pure 1-3 was confirmed using multinuclear $\left({ }^{1} \mathrm{H},{ }^{13} \mathrm{C},{ }^{29} \mathrm{Si}\right) \mathrm{NMR}$, FT-IR and HR-MS, and their size was determined by DLS and TEM-EDS analyses (for details see $\mathrm{ESI} \dagger$ ).

\subsection{Attachment of drug molecules}

As mentioned-above, due to the biocompatibility, low toxicity and inertness of silica-based materials, such compounds/ composites are successfully used in medicine. This group includes polyhedral oligomeric silsesquioxanes, which possess a three-dimensional, cage-like structure that is able to form building blocks containing an inorganic inner siloxane core. ${ }^{7}$ In addition, external terminal groups are able to bind small drug molecules, reducing their toxicity, which may be due to the sudden discharge of large amounts of the drug to the human body, and effectively assist in controlling the release. Thanks to the fact that POSS molecules can be suitably functionalized, it is possible to specify and optimize the process of the transportation of the drug, i.e. control the dose, finding respective receptors and the release of the drug from sizable organic arms. Moreover, such POSS carriers are stable in a broad range of thermal and chemical conditions, which strongly affects their in vivo stability and potential use. ${ }^{27}$

The structures of both the carrier molecules and drug molecules freely allow the attachment at the interface of pharmacologically active compounds and the network's POSS sidearms. This may be effected by hydrogen bond or halogen/ phenyl supramolecular interactions (Fig. 3).

Compound 3 was obtained in crystalline form and identified by elemental analysis, spectroscopic data, and X-ray diffraction studies. Unfortunately, its X-ray structure could not be finely resolved due to low-quality crystals. Nonetheless, thanks to our experience regarding the molecular structures of functionalized

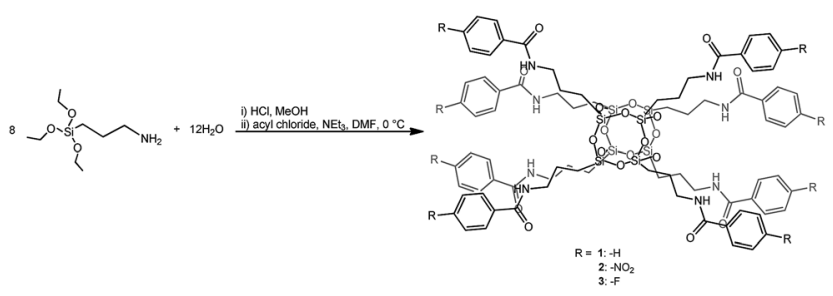

Scheme 1 Synthesis of benzamido-derivatives of octaaminopropylsilsesquioxanes 1-3. 1: benzoyl chloride; 2: 4-nitrobenzoyl chloride; 3: 4-fluorobenzoyl chloride. 
POSS, ${ }^{7}$ its structure could be clearly visualized and can be discussed. The central core of $\mathbf{3}$ is composed of eight silicon atoms forming a cubic $\mathrm{Si}_{8} \mathrm{O}_{12}$ core with eight benzamide groups attached to each silicon atom through $n$-propyl chains. An overall view of the molecule is presented in Fig. 3.

The attachment of the drug molecules process involves mixing of 1-3 as solids with ethanol solution of a drug such as ibuprofen or acetaminophen. For this purpose, the drug solution was added to a known amount of amido-functionalized POSS (for details see ESI $†$ ). The concentration of the drug in this solution was eighty times higher than the concentration of 1-3, which resulted in a tenfold excess for each side chain of the silicon cage. The resulting suspension was stirred for 2 days at $37{ }^{\circ} \mathrm{C}$. Then, the suspension was centrifuged, the precipitate was washed with ethanol, dried, and used for further studies. The resulting conjugates (POSS-drug systems) were divided into two parts: (i) the first one was used for spectroscopic studies including NMR, TG-DTA, DSC and FT-IR to examine drug attachment, and (ii) the second one was used for drug release studies verified by electronic absorption spectroscopy.

3.2.1 Attaching acetaminophen to POSS nanocarriers. NMR spectroscopy constituted one of the methods to control drug attachment to POSS compounds. In this manner, we measured the spectra of POSS-drug conjugates (1-acetaminophen; 2-acetaminophen; 3-acetaminophen) (see ESI; Fig. S1 and S2†) in DMSO- $\mathrm{d}_{6}$. Each NMR spectrum clearly confirmed the presence of attached drug molecules. Moreover, under the conditions in which the attaching process occurs, POSS molecules are stable and no cage reorganization is observed. Fig. 4 shows the NMR spectrum of a 1-acetaminophen conjugate in DMSO-d $\mathrm{d}_{6}$. The black signals derive from POSS and both the values of the chemical shifts and their multiplicity match cage-like silsesquioxane. The orange signals derive from "unchanged" drug molecules and they confirm the physical adsorption phenomenon, whereby drug molecules settle down from the solution to the carrier in a solid state.

Nanoparticle entrapment efficiency was measured by the indirect method, ${ }^{28}$ which describes the amount of the drug adsorbed to the material. This indicator makes it possible to specify and control the amount of drug molecules attached to the carrier. For 1, this value equals $64 \%$, and for 2 and 3, 39\%.

The FT-IR spectra measured in the solid state confirmed the presence of acetaminophen in the POSS conjugate. The spectra show characteristic vibrational bands derived from

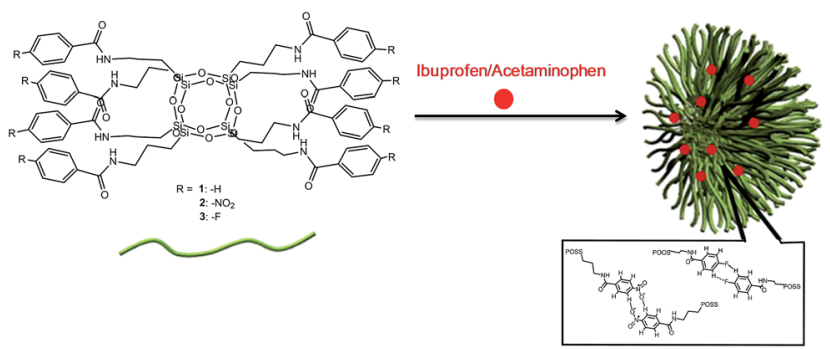

Fig. 3 Molecular structure of 3 and the idea of the attachment of drug molecules to the POSS carrier.

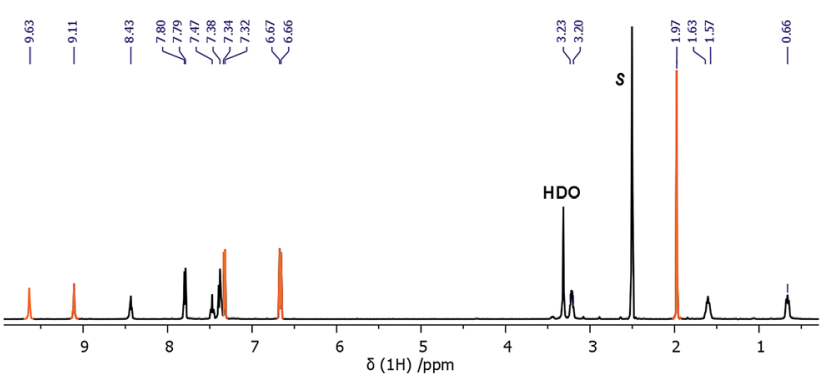

Fig. $4{ }^{1} \mathrm{H}$ NMR $\left(500 \mathrm{MHz}\right.$, DMSO- $\left.\mathrm{d}_{6}, 20^{\circ} \mathrm{C}\right)$ spectrum of $1-$ acetaminophen, $\mathrm{s}=$ solvent, orange color $=$ acetaminophen.

acetaminophen and POSS (see ESI; Fig. S3 and S5†). The attachment of acetaminophen to amide-POSS affects the reinforcement and containment of the drug within the functionalized POSS arms. To confirm the appearance of a hydrogen bond, DSC analysis was applied, because acetaminophen receives different polymorphic forms: I, II and III (see ESI; Fig. S9†). Theoretically, such a bond should simply "freeze" drug molecules in one of the mentioned-above forms. A comparison of the DSC curves indicates a confirmation of the postulated thesis, because significant differences between the graphs of the pure drug (see ESI; Fig. S9†) and POSS-drug conjugates (see ESI; Fig. S10-S12†) can be seen and can indirectly indicate the formation of a bond in the drug/carrier system. These changes are associated with a reduction in the number of exothermic peaks in the first and second heat/cooling cycles. The endothermic peak in the $163-166{ }^{\circ} \mathrm{C}$ range is associated with the melting of acetaminophen. During the second heat cycle, there are no exothermic peaks at 80 or $130{ }^{\circ} \mathrm{C}$, which confirms the lack of transition to non-active forms I and III. Using TG-DTA analysis, we also confirmed the stability of the resulting conjugates. In all cases drug-POSS systems are stable at the temperature of the human body.

3.2.2 Attaching ibuprofen to a POSS nanocarrier. Based on NMR and FT-IR spectra, it can be concluded that ibuprofen attached to amide-POSS 1-3 (see Fig. 5 and ESI; Fig. S17-S22†). The NMR spectra of the conjugates indicate the presence of chemical shifts that differ from signals typical for pure drug molecules, but it should be noted that the signals are slightly shifted. The signal integration points join a specific number of drug molecules to cage-like silsesquioxanes. In the case of $\mathbf{1}$, there are 9 drug molecules, and for 2 and 3 , there are 10 and 5 drug molecules per carrier, respectively. The DSC profiles indicate that there are small energetic changes after the attachment of the drug molecules to POSS (see ESI; Fig. S27-S29†). In case of ibuprofen, the nanoparticle entrapment efficiency is lower than that observed for acetaminophen. For 1 , this value equals $63 \%$, and for 2 and 3 it is $39 \%$.

\subsection{TEM and powder XRD analysis}

The morphology of the resulting conjugates was further studied by TEM-EDS analysis as shown in Fig. 6 .

As can be seen, amide-POSS before drug attachment possess a spherical morphology and the particles are of submicrometer 


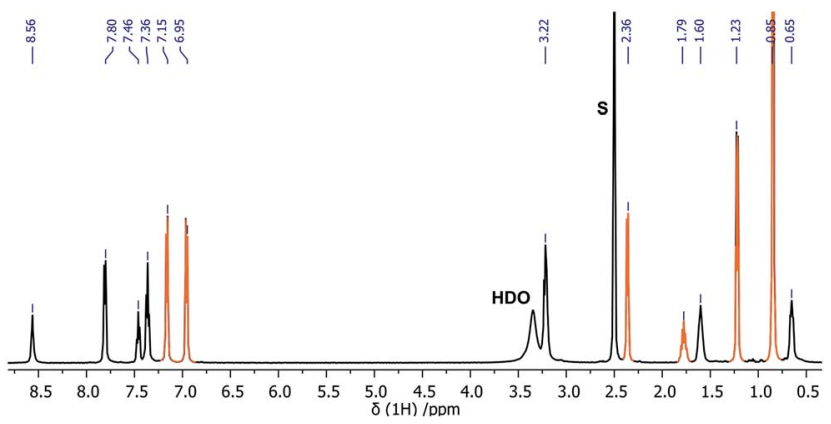

Fig. $5{ }^{1} \mathrm{H}$ NMR $\left(500 \mathrm{MHz}\right.$, DMSO- $\left.\mathrm{d}_{6}, 20^{\circ} \mathrm{C}\right)$ spectrum of 1 ibuprofen, $\mathrm{s}=$ solvent, orange color $=$ ibuprofen.

size (5-10 nm). ${ }^{26}$ POSS-drug conjugates, in turn, have a strong tendency to aggregate and, furthermore, after drugs were attached to the siloxane core via specific bonds/interactions, the mean size of the conjugates increased. Based on DLS studies, the average size of resulting drug/POSS conjugates is in the range 500-1300 nm. Fig. 6 shows the morphology of the aggregated conjugate nanoparticles in TEM (b, c, e, f, i). Their morphology changed, and the drug delivery systems formed cylindrical-like shapes as best seen in picture (h). It implied that the drug attachment would lead to the self-assembly of conjugates due to interactions between the drug molecules and the organic side-chains of the amide-POSS supports. The TEM images were the direct proof demonstrating that the drugs strongly affected the morphology of spherical-like wellseparated amide-POSS within the cylindrical drug-POSS conjugates. Additional EDS analysis performed on the conjugates confirmed that drug molecules were present in the resulting delivery systems (see ESI; Fig. S23-S25†).
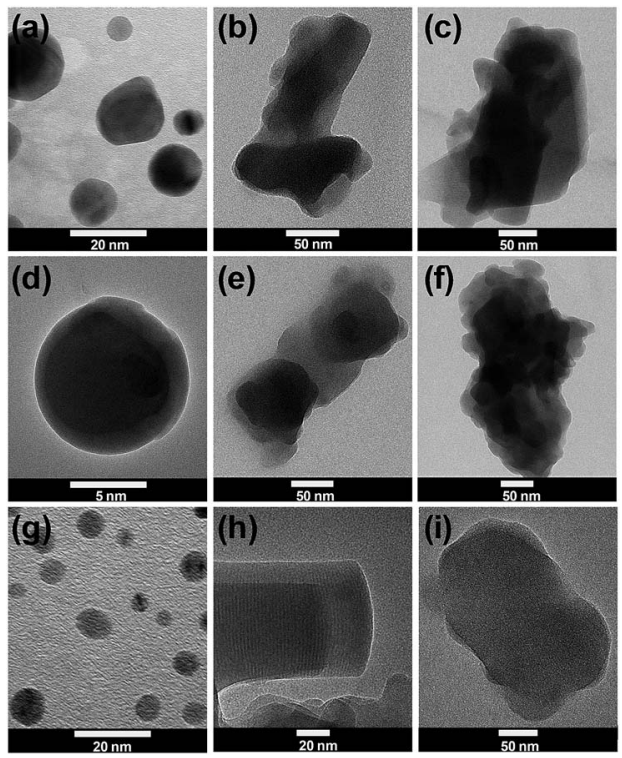

Fig. 6 Selected HR-TEM images of (a) 1, (b) 1-acetaminophen, (c) 1ibuprofen, (d) 2, (e) 2-acetaminophen, (f) 2-ibuprofen, (g) 3, (h) 3acetaminophen and (i) 3-ibuprofen particles.
Fig. 7 shows a sample of the powder X-ray diffractograms of 3 and 3 -acetaminophen in the scan angle region of $10-50^{\circ}$ (see also ESI; Fig. S34, $\dagger$ which shows PXRD patterns of 2 and 2ibuprofen). The sharp peaks at $2 \theta=16,23$ and $27^{\circ}$ correspond to the presence of drug molecules bounded to the silsesquioxane carrier. PXRD patterns confirmed the crystallinity of POSS compounds.

\subsection{Release of drug molecules}

3.4.1 Release of acetaminophen. The release process was studied by UV-Vis spectroscopy. In all samples, the mass of the released drug was calculated based on the calibration curve. It can be seen (Fig. 8) that most of the drug was released from 1 (ca. 45\%; 40 mg), whereas 2 and 3 are characterized by similar values (ca. 18\%; $\sim 13 \mathrm{mg}$ and $\sim 11 \mathrm{mg}$ for 2 - and 3-acetaminophen, respectively) of the drug released mass. Conjugate 1 released much more drug compared to 2 and 3 , which can be explained by weaker interactions with POSS in the case of 2 and $\mathbf{3}$ conjugates. The organic side chain in $\mathbf{1}$ has no substituents on the phenyl group, and there are no additional bonds that might hold drug molecules; to release the drug only hydrogen bonds needs to be broken. Compounds 2 and 3 possess additional groups on the aromatic ring, such as $-\mathrm{NO}_{2}$ and $-\mathrm{F}$, respectively, which are able to form extra interactions, like halogen bonds, or $\mathrm{C}-\mathrm{H} \cdots \mathrm{O}$ or $\mathrm{N} \cdots \mathrm{O}$ short interactions, what results in similar amounts of drug molecules released from the nanocarrier.

3.4.2 Release of ibuprofen. The release profiles for ibuprofen were monitored using the same protocol that was applied in case of acetaminophen. For ibuprofen, the biggest amount of the drug was adsorbed and released from $\mathbf{1}$, and the smallest quantities were observed for 3 (Fig. 9).

This may be due to the fact that, in case of 1-ibuprofen conjugate, the interactions between the rings are weaker due to the lack of any substituents on the phenyl ring. Moreover, the $\pi-\pi$ interaction for ibuprofen can also coexist with hydrogen bonding. The tendency to release ibuprofen is similar to that noticed for acetaminophen, i.e. it is the biggest for 1-ibuprofen, ca. $45 \%$ (ca. $40 \mathrm{mg}$ ) of the nanocarrier mass, and 35\% (ca. $24 \mathrm{mg}$ and $21 \mathrm{mg}$ for 2 - and 3-ibuprofen, respectively) for 2- and 3ibuprofen.

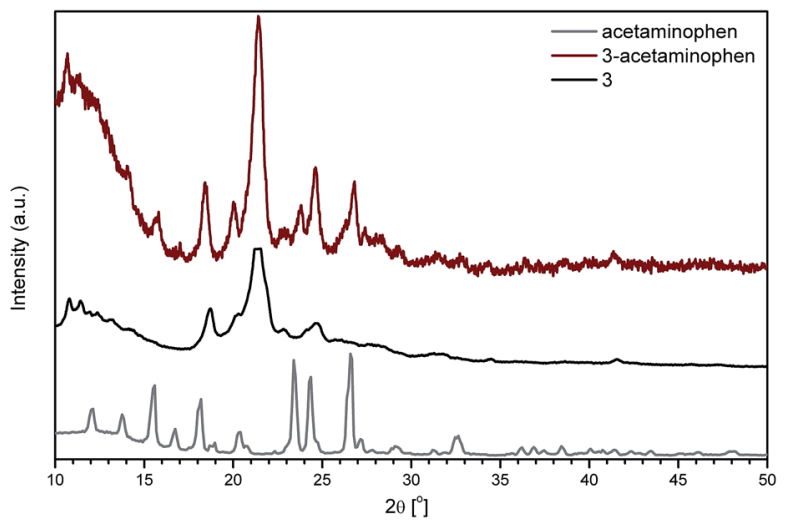

Fig. 7 Powder XRD patterns of 3 and 3-acetaminophen and acetaminophen (COD ID 2300455). 


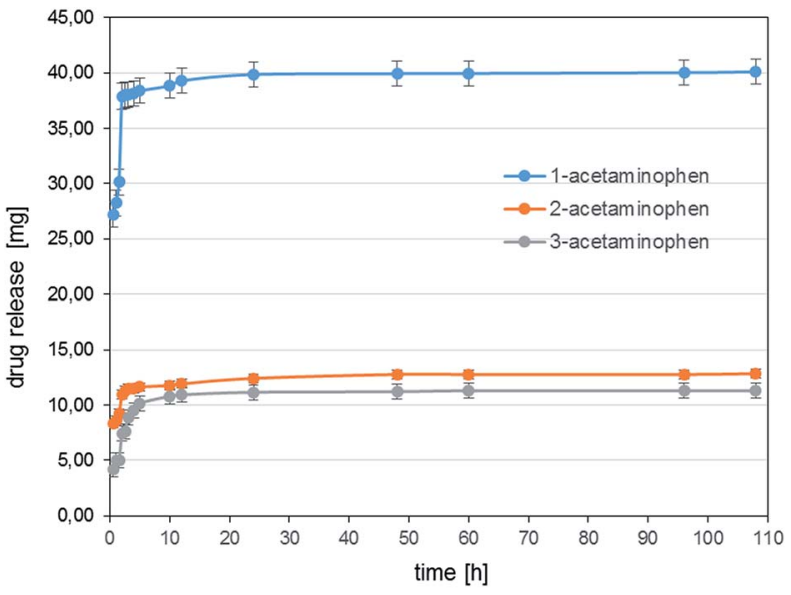

Fig. 8 Time dependence of the mass of the drug released for acetaminophen.

The release graphs for both drugs (acetaminophen and ibuprofen), which are logarithmic curves, seem typical for nanoporous and nanotubular structures. ${ }^{29}$ Furthermore, from the comparison of the presented in this paper DDS to wellstudied systems such as ramified hyperbranched polymers and dendrimers derived from POSS carriers, amide-POSS-based conjugates seems efficient. For instance, drug-loaded POSS- $g$ (PBLA $_{15}-b$-PEG) and POSS- $g$-(PBLA $30-b$-PEG) systems showed a burst release in the first $10 \mathrm{~h}$ and about $20 \%$ of quercetin was released at $\mathrm{pH}=7.4 .^{13}$

\subsection{In vitro nanoparticles degradation}

POSS nanocarriers release drugs they contain in acidic, neutral and basic environments. At $\mathrm{pH}=4$ and 7 only released drug molecules are observed, whereas at $\mathrm{pH}=7.4$ (normal tissue has a pH of around 7.40 $)^{30}$ hydrolysis of amide bond occurs. For instance, in the case of 1-ibuprofen, after $48 \mathrm{~h}$ free benzoic acid was also detected in solution, which was not observed in neutral or acidic environments. After drug release, the

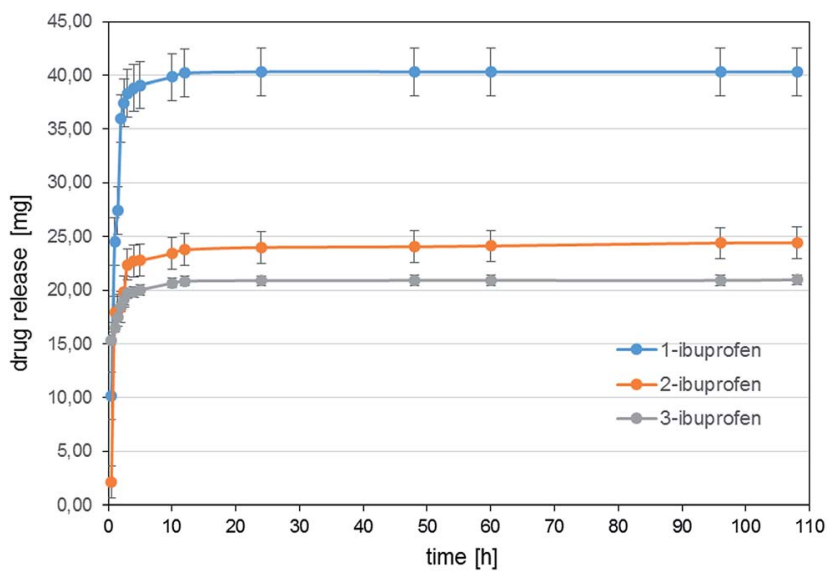

Fig. 9 Time dependence of the mass of the drug released for ibuprofen.

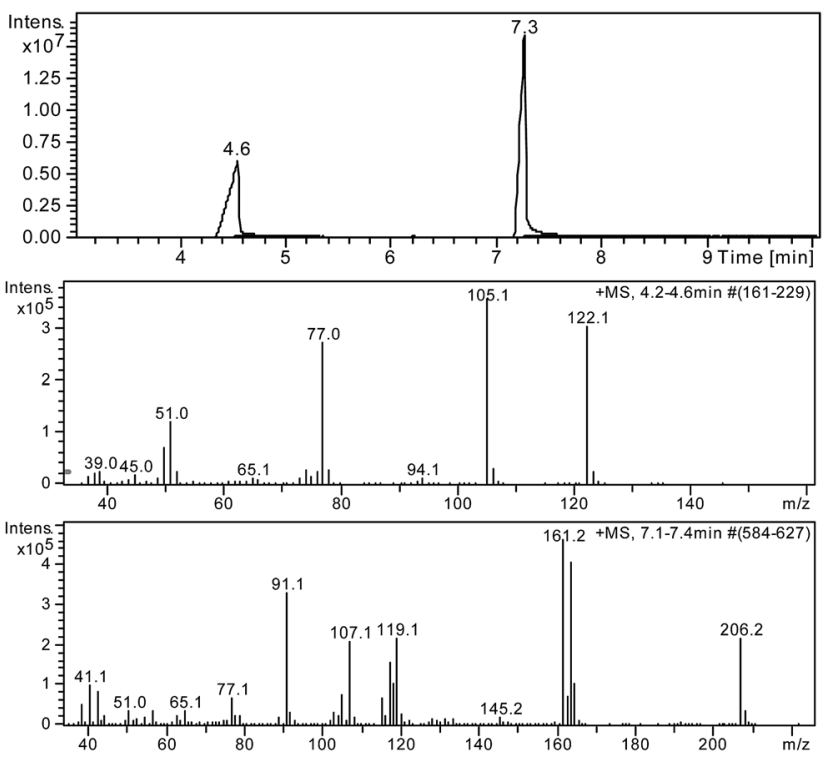

Fig. 10 Gas chromatogram and MS diagrams for 1-ibuprofen after drug release and identified at $\mathrm{pH} 7.4$ (after $48 \mathrm{~h}$ ) products.

resulting solution was examined by GC-MS, and, based on that, the proper carboxylic acids were identified by mass spectrometry. For example, Fig. 10 shows the chromatogram for 1-ibuprofen after removing the drug molecules $(\mathrm{pH}=7.4)$ from the carrier nanoparticles, where free benzoic acid was detected in solution.

\section{Conclusions}

Although the literature reports on various drug delivery systems, many synthetic laboratories still conduct studies towards the development of ideal DDSs, avoiding toxicity, low stability, low efficiency, and materials possessing high medical expectations. This sets new directions of research aimed the miniaturization of delivery systems to the nanoscale what offers many various possibilities of binding pharmacologically active particles by conjugates with the advantages of nanomaterials. In this paper, we have shown that amidofunctionalized cage-like silsesquioxanes can serve as efficient drug delivery systems. Due to the fact that such systems can be well-designed, such nanoparticles are promising drug carriers due to their ability to penetrate biological barriers such as cell membranes, which makes it possible to continue the release of the drug within the cell. In this work, we have developed a unique approach to attaching and release of drug molecules such as acetaminophen and ibuprofen, but it should be noted that amide-POSS-based systems can be utilized for the trapping of other pharmacologically active compounds if needed. In such a system, the adsorbed drug molecules can be released under physiological conditions, and then the POSS-based carrier is able to hydrolyze (at $\mathrm{pH}=7.40$ ) to non-toxic carboxylic acid salts and water soluble polyhedral oligomeric silsesquioxanes containing aminopropyl groups which products can be safely removed from the organism. 


\section{Acknowledgements}

The authors would like to express their gratitude to the National Science Centre in Poland for financial support (Grant No. 2013/ 09/D/ST8/03995).

\section{References}

1 Fundamentals and Applications of Controlled Release Drug Delivery, ed. J. Siepmann, R. A. Siegel and M. J. Rathbone, Springer, US, Boston, MA, 2012.

2 G. M. Whitesides and B. Grzybowski, Science, 2002, 295, 2418-2421.

3 D. A. Tomalia, J. B. Christensen and U. Boas, Dendrimers, Dendrons, and Dendritic Polymers: Discovery, Applications, and the Future, Camb. Univ. Press, 2012.

4 W.-B. Zhang, X. Yu, C.-L. Wang, H.-J. Sun, I.-F. Hsieh, Y. Li, X.-H. Dong, K. Yue, R. Van Horn and S. Z. D. Cheng, Macromolecules, 2014, 47, 1221-1239.

5 W.-B. Zhang and S. Z. D. Cheng, Chin. J. Polym. Sci., 2015, 33, 797-814.

6 M. Huang, C.-H. Hsu, J. Wang, S. Mei, X. Dong, Y. Li, M. Li, H. Liu, W. Zhang, T. Aida, W.-B. Zhang, K. Yue and S. Z. D. Cheng, Science, 2015, 348, 424-428.

7 M. Janeta, Ł. John, J. Ejfler, T. Lis and S. Szafert, Dalton Trans., 2016, 45, 12312-12321.

8 M. Hamidi, A. Azadi and P. Rafiei, Adv. Drug Delivery Rev., 2008, 60, 1638-1649.

9 T. M. Allen and P. R. Cullis, Science, 2004, 303, 1818-1822.

10 N. Sanvicens and M. P. Marco, Trends Biotechnol., 2008, 26, 425-433.

11 R. H. Baney, M. Itoh, A. Sakakibara and T. Suzuki, Chem. Rev., 1995, 95, 1409-1430.

12 C. McCusker, J. B. Carroll and V. M. Rotello, Chem. Commun., 2005, 996-998.

13 Y. Pu, L. Zhang, H. Zheng, B. He and Z. Gu, Macromol. Biosci., 2014, 14, 289-297.

14 T. L. Kaneshiro and Z.-R. Lu, Biomaterials, 2009, 30, 56605666.
15 A. Janaszewska, K. Gradzinska, M. Marcinkowska, B. Klajnert-Maculewicz and W. A. Stanczyk, Materials, 2015, 8, 6062-6070.

16 H. J. Erli, R. Marx, O. Paar, F. U. Niethard, M. Weber and D. C. Wirtz, Biomed. Eng. Online, 2003, 2, 15.

17 F. Yan, H. Xu, J. Anker, R. Kopelman, B. Ross, A. Rehemtulla and R. Reddy, J. Nanosci. Nanotechnol., 2004, 4, 72-76.

18 S.-G. Zhu, J.-J. Xiang, X.-L. Li, S.-R. Shen, H. Lu, J. Zhou, W. Xiong, B.-C. Zhang, X.-M. Nie, M. Zhou, K. Tang and G.-Y. Li, Biotechnol. Appl. Biochem., 2004, 39, 179-187.

19 P. Kortesuo, M. Ahola, M. Kangas, T. Leino, S. Laakso, L. Vuorilehto, A. Yli-Urpo, J. Kiesvaara and M. Marvola, J. Controlled Release, 2001, 76, 227-238.

20 J.-F. Chen, H.-M. Ding, J.-X. Wang and L. Shao, Biomaterials, 2004, 25, 723-727.

21 M. A. Hood, M. Mari and R. Muñoz-Espí, Materials, 2014, 7, 4057-4087.

22 Long Acting Injections and Implants, ed. J. C. Wright and D. J. Burgess, Springer, US, Boston, MA, 2012.

23 G. Kwon, M. Naito, M. Yokoyama, T. Okano, Y. Sakurai and K. Kataoka, Langmuir, 1993, 9, 945-949.

24 Ł. John, M. Podgórska, J.-M. Nedelec, Ł. Cwynar-Zajac and P. Dzięgiel, Mater. Sci. Eng., C, 2016, 68, 117-127.

25 Ł. John, M. Bałtrukiewicz, P. Sobota, R. Brykner, Ł. CwynarZając and P. Dzięgiel, Mater. Sci. Eng., C, 2012, 32, 18491858.

26 M. Janeta, Ł. John, J. Ejfler and S. Szafert, Chem.-Eur. J., 2014, 20, 15966-15974.

27 P. Torricelli, E. Verné, C. V. Brovarone, P. Appendino, F. Rustichelli, A. Krajewski, A. Ravaglioli, G. Pierini, M. Fini, G. Giavaresi and R. Giardino, Biomaterials, 2001, 22, 2535-2543.

28 S. Fattahpour, M. Shamanian, N. Tavakoli, M. Fathi, S. R. Sheykhi and S. Fattahpour, J. Appl. Polym. Sci., 2015, 132, 42241.

29 M. S. Aw, M. Kurian and D. Losic, Biomater. Sci., 2013, 2, 1034.

30 W.-Y. Qian, D.-M. Sun, R.-R. Zhu, X.-L. Du, H. Liu and S.-L. Wang, Int. J. Nanomed., 2012, 7, 5781-5792. 